\title{
Feasibility, safety, electrophysiological characteristics and mid-term outcomes of Selective Left bundle branch pacing - Indian perspective
}

\author{
Shunmuga Sundaram ${ }^{1}$, Giridhar Muthu ${ }^{1}$, Mahesh Kumar ${ }^{1}$, Dasarath Boppana ${ }^{1}$, Vijesh \\ Anand $^{1}$, and Surya Kumar ${ }^{1}$ \\ ${ }^{1}$ Velammal Medical College Hospital and Research Institute
}

April 28, 2020

\begin{abstract}
Background: His bundle pacing (HBP) has evolved as the most physiological form of pacing but associated with limitations. Recently left bundle branch pacing (LBBP) is emerging as an effective alternative strategy for HBP. Objectives: Our study was designed to assess the feasibility, efficacy, electrophysiological parameters and mid-term outcomes of LBBP in Indian population Methods: All patients requiring permanent pacemaker implantation for symptomatic bradycardia and heart failure were prospectively enrolled. Echocardiography, QRS duration, pacing parameters, Left bundle(LB) potentials, paced QRS duration and peak left ventricular activation time (pLVAT) recorded. Results: LBBP was successful in 93 out of 99 patients (94\% acute success). Mean age $62.6 \pm 13$ yrs. Male 59\%, diabetes $69 \%$, coronary artery disease $65 \%$. Follow up duration 4.8 months (range1-12 months). Indication for pacing were atrioventricular (AV) block 43\%, cardiac resynchronization therapy 40\%, AV node ablation 4\%. LB potential noted in 37 patients (40\%). QRS duration reduced from $144.38 \pm 34.6 \mathrm{~ms}$ at baseline to $110.8 \pm 12.4 \mathrm{~ms}$ after LBBP (p value 0.0001). Pacing threshold was $0.59 \pm 0.22 \mathrm{~V}$ and sensed $\mathrm{R}$ wave $14.14 \pm 7.19 \mathrm{mV}$ and it remained stable during follow up. Lead depth in the septum was $9.62 \mathrm{~mm}$. LV ejection fraction increased from $44.96 \%$ to $53.3 \%$ after LBBP (p value 0.0001). One died due to respiratory tract infection on follow up Conclusion: LBBP is a safe and effective strategy (94\% acute success) of physiological pacing. The pacing parameters remained stable over a period of 12 months follow up. LBBP can effectively overcome the limitations of HBP
\end{abstract}

\section{Hosted file}

Article LBBP - Indian study.doc available at https://authorea.com/users/309818/articles/440687feasibility-safety-electrophysiological-characteristics-and-mid-term-outcomes-of-selectiveleft-bundle-branch-pacing-indian-perspective

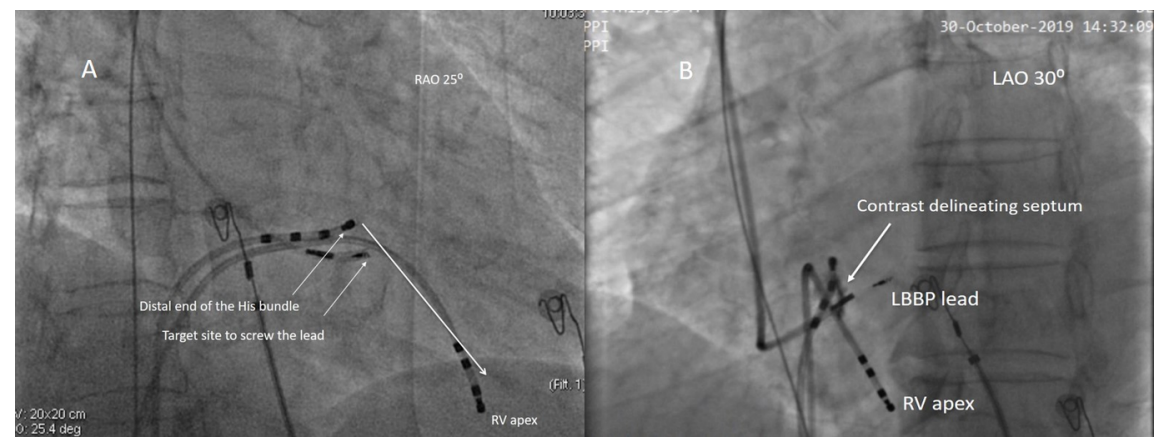



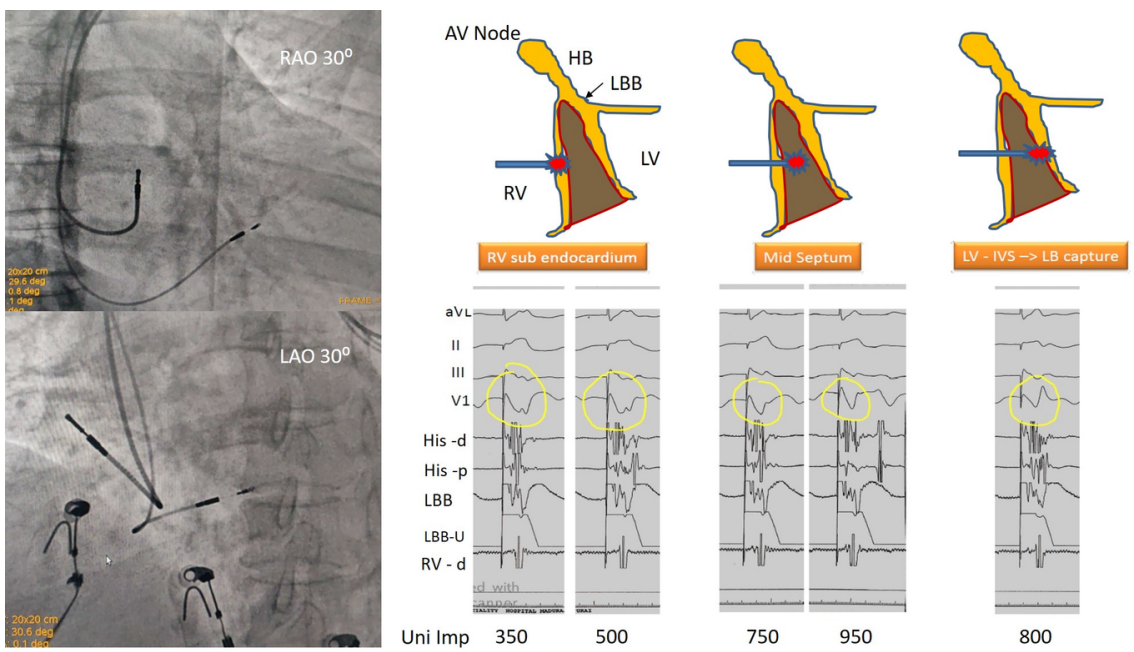

800

- Quadripolar His catheter from FV $\rightarrow$ Quick mapping to know the distal extent

- 3830 lead with C315 sheath $\rightarrow 1-1.5 \mathrm{~cm}$ below the His catheter along the imaginary line in RAO view

- LAO $30^{\circ}$ View $\rightarrow$ Rapid 4-5 turns monitoring the lead tip - based on septal thickness

- Measure QRS, unipolar impedance ( $\geq 500$ ohms), $\operatorname{pLVAT}(<75-80 \mathrm{~ms})$

- Contrast Angiography using $2-3 \mathrm{ml}$ in LAO $30^{\circ}$ View to assess the lead depth

- Peel away the sheath
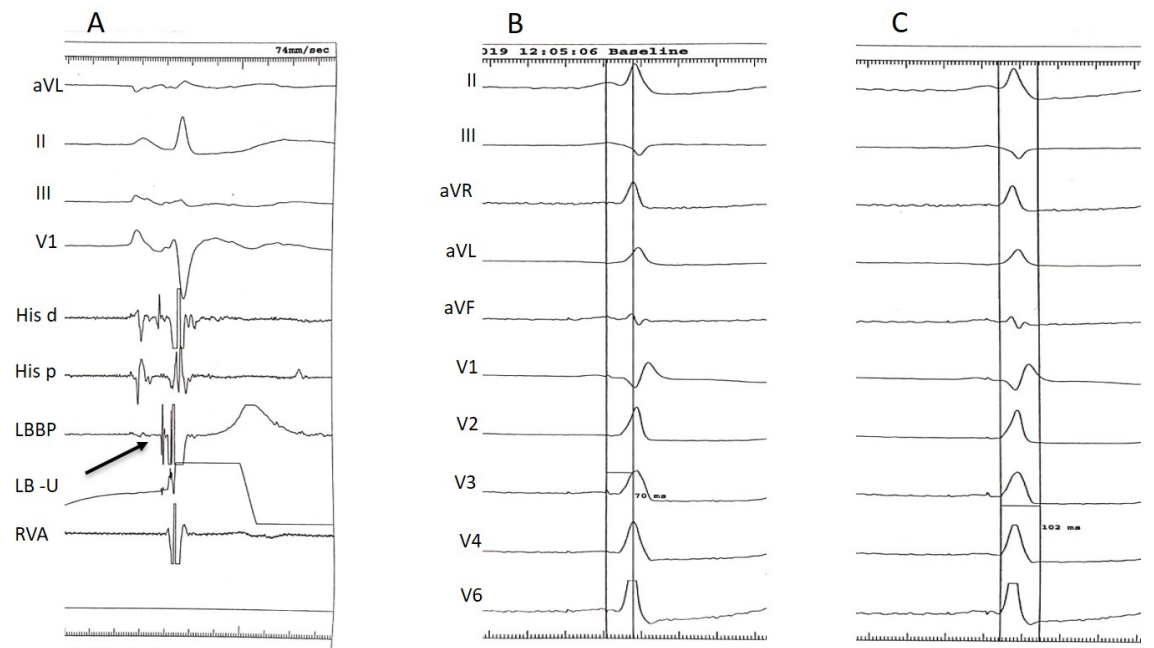

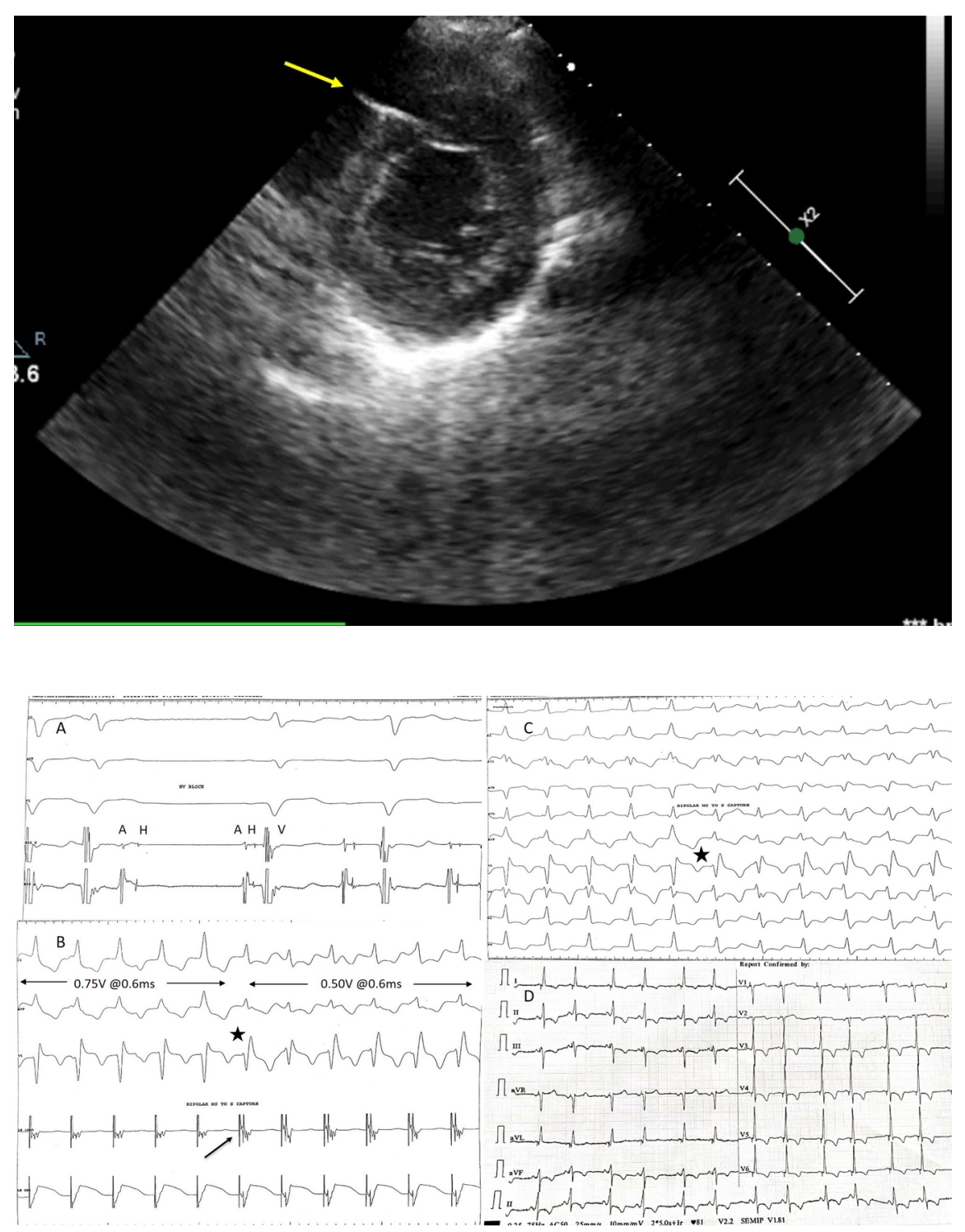


\begin{tabular}{|c|c|}
\hline Total number of Patients & 99 patients \\
\hline Successful LBB Pacing & 93 Patients (94\%) \\
\hline Follow up (months) & 4.8 months(range $1-12$ months) \\
\hline Age (yrs) & $62.6 \pm 13.1$ \\
\hline $\begin{array}{l}\text { Men } \\
\text { Women }\end{array}$ & $\begin{array}{l}55 \text { (59\%) } \\
38(41 \%)\end{array}$ \\
\hline $\begin{array}{l}\text { Hypertension } \\
\text { Diabetes Mellitus } \\
\text { Coronary artery disease } \\
\text { Atrial fibrillation } \\
\text { LV dysfunction (EF }<50 \% \text { ) }\end{array}$ & $\begin{array}{l}61 \% \\
69 \% \\
65 \% \\
6 \% \\
58.1 \%\end{array}$ \\
\hline $\begin{array}{l}\text { Pacing Inducations } \\
\text { Sick sinus Syndrome } \\
\text { AV block } \\
\text { Cardiac resynchronisation therapy } \\
\text { Atrial fibrillation with FVR/AVJ ablation } \\
\text { Pacing induced Cardiomyopathy }\end{array}$ & $\begin{array}{l}8(9 \%) \\
40(43 \%) \\
37(40 \%) \\
4(4 \%) \\
4(4 \%)\end{array}$ \\
\hline $\begin{array}{l}\text { Procedure Characteristics } \\
\text { LBBP fluoroscopy time (mins) } \\
\text { Total fluoroscopy time (mins) } \\
\text { Sheath Angiography }\end{array}$ & $\begin{array}{l}22.94 \pm 11.7 \\
28.59 \pm 13.3 \\
56 \text { Patients }(61 \%)\end{array}$ \\
\hline $\begin{array}{l}\text { Baseline ECG } \\
\text { QRS duration } \\
\text { LBBB morphology } \\
\text { RBBB morphology } \\
\text { IVCD }\end{array}$ & $\begin{array}{l}144.38 \pm 34.6 \mathrm{~ms} \\
38 \\
12 \\
7\end{array}$ \\
\hline
\end{tabular}




\begin{tabular}{|c|c|}
\hline $\begin{array}{l}\text { Electrophysiology parameters } \\
\text { Left bundle potential } \\
\text { LB potential -QRS duration (ms) } \\
\text { LB paced QRS duration (ms) } \\
\text { pLVAT (ms) }\end{array}$ & $\begin{array}{l}37 \text { patients }(40 \%) \\
24.9 \pm 0.49 \mathrm{~ms} \\
110.8 \pm 12.4 \mathrm{~ms} \\
72.5 \pm 10.8 \mathrm{~ms}\end{array}$ \\
\hline $\begin{array}{l}\text { Pacing Parameters } \\
\text { Threshold (unipolar) @0.6ms PW } \\
\text { Anodal threshold @0.6ms PW } \\
\text { Sensed R wave (mV) } \\
\text { Unipolar pacing impedance (ohms) }\end{array}$ & $\begin{array}{l}0.59 \pm 0.22 \mathrm{~V} \\
2.02 \pm 0.3 \mathrm{~V} \\
14.14 \pm 7.19 \mathrm{mV} \\
679.4 \pm 123.7 \mathrm{ohms}\end{array}$ \\
\hline $\begin{array}{l}\text { Echocardiographic Parameters } \\
\text { Baseline LVEF (\%) } \\
\text { Septal Thickness (mm) } \\
\text { Lead depth (mm) } \\
\text { Worsening of LVEF }\end{array}$ & $\begin{array}{l}44.96 \pm 14.6 \% \\
10.73 \pm 1.56 \mathrm{~mm} \\
9.62 \pm 1.01 \mathrm{~mm} \\
\text { Nil }\end{array}$ \\
\hline $\begin{array}{l}\text { Safety Parameters } \\
\text { Acute Lead dislodgement } \\
\text { Late lead dislodgement } \\
\text { Late raise in threshold by }>1 \mathrm{~V} \\
\text { Thromboembolic complication } \\
\text { Mortality }\end{array}$ & $\begin{array}{l}\text { Nil } \\
\text { Nil } \\
\text { Nil } \\
\text { Nil } \\
1 \text { (non cardiac) }\end{array}$ \\
\hline
\end{tabular}

\begin{tabular}{|l|l|l|l|}
\hline & At implantation & Follow up (1-12 months) & P Value \\
\hline $\begin{array}{l}\text { Pacing Parameters } \\
\text { Threshold (Unipolar) } \\
\text { R wave (mV) }\end{array}$ & $0.59 \pm 0.22 \mathrm{~V}$ & $0.57 \pm 0.12 \mathrm{~V}$ & 0.245 \\
$\begin{array}{l}\text { Pacing Impedance (ohms) } \\
\text { ECG - QRS duration (Pre and Post) }\end{array}$ & $\begin{array}{l}14.14 \pm 7.19 \mathrm{mV} \\
679.4 \pm 123.7 \mathrm{ohms}\end{array}$ & $\begin{array}{l}13.68 \pm 5.2 \mathrm{mV} \\
607.7 \pm 83.5 \mathrm{ohms}\end{array}$ & 0.199 \\
\hline $\begin{array}{l}\text { Echocardiographic Paraemters } \\
\text { LV ejection Fraction }\end{array}$ & $144.38 \pm 34.6 \mathrm{~ms}$ & $110.8 \pm 12.4 \mathrm{~ms}$ & 0.0012 \\
\hline Worsening of Tricuspid regurgitation & $44.96 \pm 14.6 \%$ & $53.3 \pm 10.9 \%$ & 0.0001 \\
\hline & -- & Nil & 0.0001 \\
\hline
\end{tabular}

